\title{
RELAÇÕES FISIOECOLÓGICAS NO DIPLÓPODO Oxidus gracilis (POLYDESMIDA, STRONGYLOSOMIDADE): 0 CONSUMO DE OXIGÊNIO EM RELAÇÃO AO TAMANHO, SEXO E CONDIÇÃO ALIMENTAR
}

\section{RICARDO SANTOS DA SILVA ${ }^{1}$, CARLOS HENRIQUE SILVA PENTEADO $^{2}$ E LILIAN BOCCARDO ${ }^{3}$}

${ }^{1}$ Departamento de Zoologia - UNESP - Campus de Rio Claro

${ }^{2}$ Centro de Estudos Ambientais (CEA) - UNESP - Campus de Rio Claro

${ }^{3}$ Departamento de Ciências Biológicas - UESB - Campus de Jequié - BA.

\section{RESUMO}

O consumo de oxigênio do diplópodo Oxidus gracilis foi determinado em respirômetro de Warburg, a $25^{\circ} \mathrm{C}$, em 21 machos e 24 fêmeas, com a finalidade de estudar as relações fisioecológicas e metabólicas com o tamanho, sexo e condição alimentar.

Expressando o metabolismo ou consumo horário de oxigênio $(\mathrm{M})$ em função da massa corpórea (P) e usando regressões logarítmicas, os resultados da equação alométrica $\mathrm{M}=\mathrm{a} \cdot \mathrm{P}^{\mathrm{b}}$, foram: $\mathrm{M}=2,589 . \mathrm{P}^{0,89}$ para os machos, $\mathrm{M}=2,715 \cdot \mathrm{P}^{0,78}$ para as fêmeas e $\mathrm{M}=2,6814 . \mathrm{P}^{0,86}$ para ambos os sexos, conjuntamente. Os coeficientes de regressão (índices de b) obtidos indicaram que o metabolismo respiratório de Oxidus gracilis fica num nível intermediário de dependência, entre a massa e a superfície corpórea.

Os efeitos da condição alimentar foram avaliados em diplópodos recémalimentados e jejunos de 24, 48, 72 e 96 horas. Ao final do $5^{\circ}$ dia $(96 \mathrm{~h})$ a massa corpórea teve uma redução de $21,46 \%$ e o consumo de oxigênio de $52,37 \%$. Em ambos os casos as reduções foram significativas $(\mathrm{P}<0,05)$. A despeito de que nenhuma morte tenha ocorrido durante o período estudado, concluiu-se que o estresse provocado pelo jejum alimentar produz mudanças mais acentuadas no metabolismo do que na massa corpórea 
de Oxidus gracilis.

Palavras-chave: Oxidus gracilis, metabolismo, tamanho, sexo, condição alimentar.

\section{ABSTRACT}

The oxygen consumption of the millipede Oxidus gracilis was determined in an Warburg respirometer at $25^{\circ} \mathrm{C}$, using males and females in order to detect the physioecologic and metabolic responses related to size, sex and alimentary conditions. Expressing metabolism (M) in function to body mass (P), and employing logarithmic data in the allometrical relationships, result of the $\mathrm{M}=\mathrm{a} . \mathrm{P}^{\mathrm{b}}$ equations, were: $\mathrm{M}=$ 2,589. $\mathrm{P}^{0,89}$ for males (21 individuals), $\mathrm{M}=2,715 . \mathrm{P}^{0,78}$ for females (24 individuals), and $\mathrm{M}=2,6814 . \mathrm{P}^{0,86}$ for males and females, conjointly. The regression coefficients $(\mathrm{b}$ indexes) showed that respiratory metabolism of Oxidus gracilis would stay in an intermediary level of dependence between body mass, and body surface.

The effects of alimentary condition were studied right before feeding, and after $24,48,72$ and 96 hours of fasting. At the end of the $5^{\text {th }}$ day (96 h), animals had a reduction of $21,46 \%$ in body mass, and $52,37 \%$ in oxygen uptake. In both cases, reductions were significant $(\mathrm{P}<0,05)$. Despite of no deaths have occurred, results indicate that stress due to abstinence from food, produce more changes in metabolism than in body mass of Oxidus gracilis.

Key-words: Oxidus gracilis, metabolism, size, sex, alimentary condition.

\section{INTRODUÇÃO}

Um aspecto importante para o estudo da fisioecologia dos animais de solo é o da determinação do metabolismo respiratório, que fornece subsídios para a compreensão da participação dos animais no fluxo de energia do solo, a "Energy Turnover" (NIELSEN, 1961; PHILLIPSON, 1966; REICHE, 1968). Todavia o estudo do metabolismo respiratório é também fundamental para a compreensão dos aspectos básicos da biologia, fisiologia, ecologia e comportamento metabólico dos animais, principalmente quando é relacionado com o tamanho, sexo, condição alimentar e outros fatores intrínsecos e extrínsecos (BOCCARDO, 1992; BOCCARDO, 1996; BRITES, 1982; PENTEADO, 1972; PROSSER, 1973).

Nos animais, as interações entre metabolismo e tamanho seguem, em geral, a equação alométrica $\mathbf{M}=\mathbf{a} . \mathbf{P}^{\mathbf{b}}$ de HUXLEY (1932), onde $\mathbf{M}$ é o metabolismo, $\mathbf{P}$ o peso 
do corpo (massa corpórea) e, a e $\mathbf{b}$, constantes que representam, respectivamente, a interseção e a inclinação da reta que se obtém, plotando-se graficamente os valores do log do peso (massa), contra o log do metabolismo ou consumo de oxigênio. Nessas condições, a dá uma idéia do nível metabólico do animal e b (coeficiente de regressão) a proporção com que o metabolismo se altera com o tamanho. Normalmente b varia de 0,66 (metabolismo proporcional à superfície do animal) até 1,00 (metabolismo proporcional à massa corpórea), o que permitiu a LUDWIG (1956), LUDWIG \& KRYWIENCZYK (1950) e BERTALANFFY (1957) classificarem os animais em diferentes padrões metabólicos de acordo com o valor do expoente $\mathbf{b}$.

Em diplópodos, as relações entre metabolismo respiratório e tamanho foram pesquisadas por autores americanos (MORRISON, 1946; REICHLE, 1968; WOOTEN \& CRAWFORD, 1974), poloneses (GROMYSZ-KALKOWSKA, 1970; 1973; 1974; 1976; 1979; GROMYSZ-KALKOWSKA \& STOJALOWSKA, 1966; 1971; 1973; GROMYSZ-KALKOWSKA et al., 1986), russos (BYZOVA, 1967; 1971), indianos (DWARAKANATH, 1971; DWARAKANATH \& JOB, 1966; DWARAKANATH, et al., 1972; 1973; PAULPANDIAN, 1966), australianos (HUMPHREYS \& COLLIS, 1990) e gregos (STAMOU \& IATROU, 1993).

No Brasil podem ser citados os trabalhos efetuados por PENTEADO (1986); PENTEADO \& MENDES (1977; 1981); PENTEADO et al. (1991) e BOCCARDO \& PENTEADO (1995) envolvendo, respectivamente, os diplópodos Plusioporus setiger, Rhinocricus padbergi, Pseudonannolene tricolor e Gymnostreptus olivaceus, cujos resultados possibilitaram estabelecer comparações metabólicas com as espécies de diferentes hábitats e regiões geográficas, bem como de tamanhos corpóreos e de condições fisiológicas diversas.

O fator sexo constitui-se num outro agente modificador do metabolismo, cujos efeitos se fazem sentir mais acentuadamente nos períodos reprodutivos (PENTEADO, 1972; SILVA, 1997). Tais períodos, todavia, normalmente são evitados pelos pesquisadores, posto que a maior sensibilidade dos animais interfere nas relações do metabolismo com o tamanho e nas interpretações de cunho comparativo. Com referência aos diplópodos, alterações metabólicas devidas ao sexo foram registradas por HALL et al. (1969), DWARAKANATH et al. (1972) e GROMYSZ-KALKOWSKA (1979).

O metabolismo respiratório também pode ser afetado pelas condições de saciedade ou de privação de alimento. Animais recém-alimentados tendem a apresentar durante os processos de digestão e absorção, um aumento do metabolismo e nos estados de jejum ou carência, uma diminuição da taxa metabólica (PROSSER, 1973; ZEUTHEN, 1947). O problema no tocante aos diplópodos foi investigado em termos respirométricos por BYZOVA (1967, 1972), GROMYSZ-KALKOWSKA (1970, 1973), GROMYSZKALKOWSKA \& STOJALOWSKA (1971), PENTEADO \& MENDES (1977) E 
STEWART \& WOODRING (1973).

No presente trabalho o metabolismo respiratório do diplópodo tropical Oxidus gracilis foi investigado em relação aos fatores intrínsecos, tamanho, sexo e estado nutricional (animais alimentados e jejunos), visando verificar o grau de dependência metabólica do animal a esses fatores.

\section{MATERIAL E MÉTODOS}

\section{Oxidus gracilis}

Oxidus gracilis (Koch, 1847) é um diplópodo tropical de vida gregária, cujos adultos atingem cerca de $40 \mathrm{~mm}$ (SCHUBART, 1955), de ocorrência durante o ano inteiro, mas escassos nos meses sem chuva e com temperaturas muito baixas no inverno (SCHUBART, 1944). No Brasil, os indivíduos jovens ocorrem, principalmente, da metade da primavera, até o final do verão. A diferenciação sexual é constatada pela presença dos gonopódios copuladores no sétimo segmento dos machos. Os exemplares utilizados neste trabalho foram coletados no jardim experimental da UNESP - Campus de Rio Claro - SP (22 $\left.24^{\prime} 36^{\prime \prime} \mathrm{S}, 47^{\circ} 33^{\prime} 36^{\prime \prime} \mathrm{W}\right)$.

\section{Metodologia}

O metabolismo respiratório de Oxidus gracilis foi pesquisado a $25{ }^{\circ} \mathrm{C}$, em respirômetro de Warburg, seguindo o método usual de respirometria (DIXON, 1943). Foram utilizados frascos de aproximadamente $20 \mathrm{ml}$ de capacidade, aos quais foram adicionados $0,3 \mathrm{ml}$ de solução de $\mathrm{KOH}$ a $14 \%$ para absorção de $\mathrm{CO}_{2}$ expirado. Nos experimentos foram usados animais em fase de intermuda, colocando-se apenas 1 diplópodo por frasco e o critério adotado foi o do metabolismo de rotina, onde os indivíduos não sofrem contenção dos movimentos dentro do sistema.

Os estudos envolvendo tamanho e sexo foram feitos em 21 machos e 24 fêmeas, jejunos de 24 horas, jovens e adultos; com massa corpórea variando de 0,041 a 0,083 g. No estudo dos efeitos da condição alimentar foi utilizado um lote de 12 indivíduos adultos recém-alimentados e submetidos a jejum de 24, 48, 72 e $96 \mathrm{~h}$ ( 5 dias). Neste caso não se levou em conta o sexo e a massa corpórea média inicial foi de $0,063 \pm$ 0,007 g. Durante o tempo de privação de alimento, os diplópodos permaneceram em câmaras climáticas a $25^{\circ} \mathrm{C}$, dentro de placas de Petri, tampadas e forradas com papel de filtro umedecido para protegê-los da desidratação. Todos os dados de consumo de oxigênio ou metabolismo foram expressos em $\mu 1 \mathrm{O}_{2} \cdot \mathrm{h}^{-1}$.

\section{RESULTADOS E DISCUSSÃO}


Confrontando-se o consumo horário de oxigênio de cada diplópodo com o seu tamanho (massa corpórea) e tratando-se os dados logaritmicamente, as equações alométricas $\mathrm{M}=\mathrm{a} \cdot \mathrm{P}^{\mathrm{b}}$ foram: $\mathrm{M}=2,589 . \mathrm{P}^{0,89}$ para os machos $\mathrm{e} \mathrm{M}=2,715 . \mathrm{P}^{0,78}$ para as fêmeas (Figuras 1 e 2).



Figura 1. Relação entre o consumo horário de oxigênio e tamanho (massa corpórea) de Oxidus gracilis (machos) a $25^{\circ} \mathrm{C} . \mathbf{b}=\mathbf{0 , 8 9} ; \mathrm{r}=0,79 ; \mathrm{P}<0,01$.

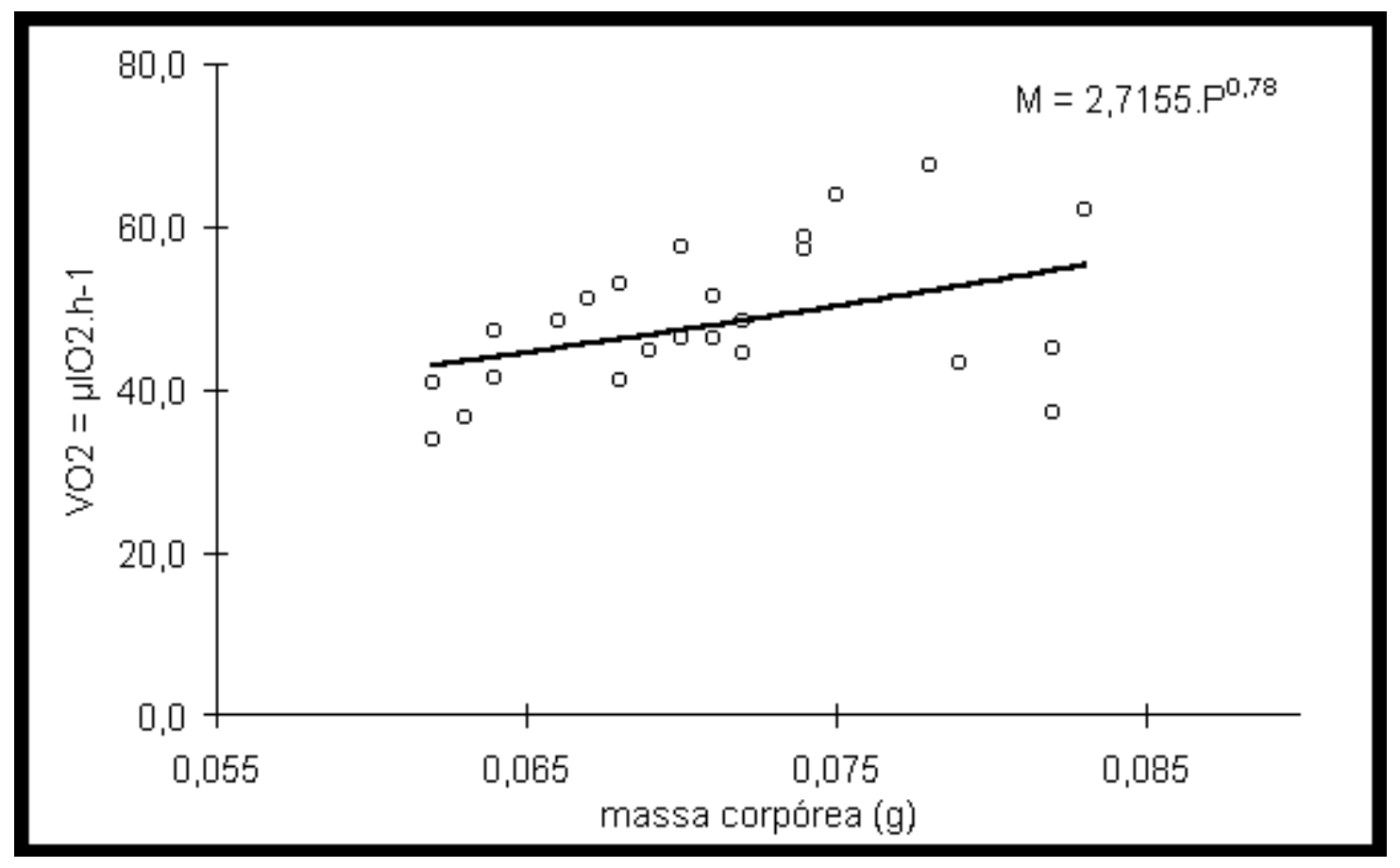

Figura 2. Relação entre o consumo horário de oxigênio e tamanho (massa corpórea) de Oxidus gracilis (fêmeas) a $25^{\circ} \mathrm{C} . \mathbf{b}=\mathbf{0 , 7 8} ; \mathrm{r}=0,82 ; \mathrm{P}<0,01$.

A possibilidade de haver, ou não, influência do sexo no metabolismo foi 
pesquisada por meio de um teste de comparação entre os 2 coeficientes de regressão (b) (BAILEY, 1959). O resultado foi não significativo $(\mathrm{P}>0,05)$, a despeito das fêmeas apresentarem massa corpórea superior a dos machos. Uma nova regressão geral incluindo machos e fêmeas foi calculada e a expressão $\mathrm{M}=2,6814 . \mathrm{P}^{0,86}$ foi admitida como representativa da relação entre metabolismo e tamanho em Oxidus gracilis, ajustando-se o metabolismo do diplópodo a um padrão intermediário entre a superfície e a massa corpórea (Figura 3).

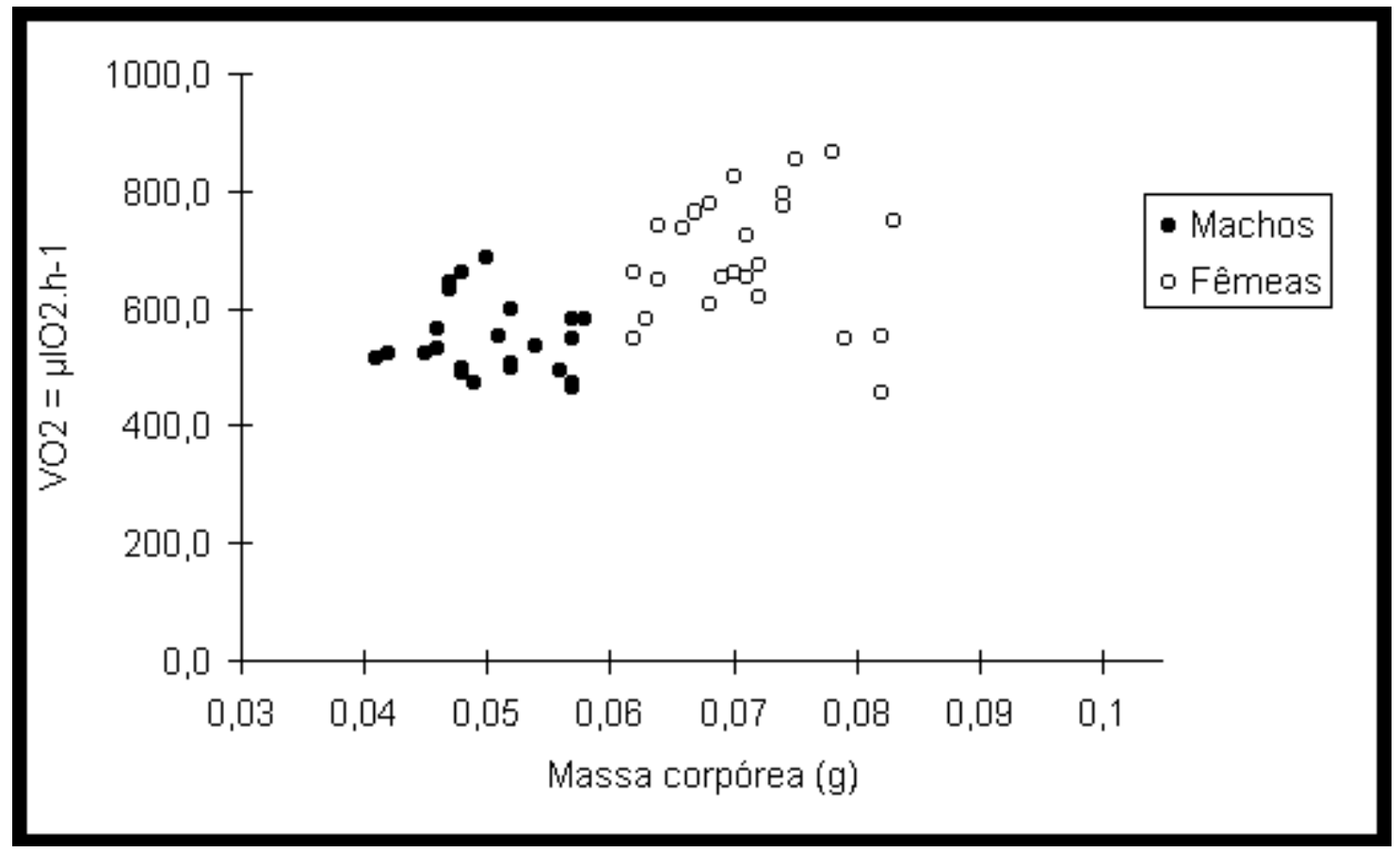

Figura 3. Relação entre o consumo horário de oxigênio e tamanho (massa corpórea) de machos e fêmeas de Oxidus gracilis, a $25^{\circ} \mathrm{C}$. ( b (comum) $\left.=\mathbf{0 , 8 6} ; \mathrm{r}=0,77 ; \mathrm{P}<0,01\right)$.

Os efeitos do jejum alimentar na perda de peso e no metabolismo de Oxidus gracilis (Tabela 1, Figura 4) ocorreram nos dois parâmetros analisados. Ao final do $5^{\circ}$ dia (96 h), a redução da massa corpórea foi de $21,46 \%$ e a do consumo de oxigênio foi de $52,37 \%$. Em ambos os casos, as reduções foram significativas $(\mathrm{P}<0,05)$ nas análises feitas por meio de testes $t$ para dados emparelhados (GRANER, 1966). A amplitude das variações sugeriu, entretanto, que o estresse produzido pelo jejum alimentar altera mais acentuadamente o metabolismo do que a massa corpórea de Oxidus gracilis. É necessário esclarecer que nenhum diplópodo morreu durante os experimentos.

Tabela 1 - Relação entre a perda de massa corpórea e o metabolismo (consumo horário de oxigênio) a $25^{\circ} \mathrm{C}$ de Oxidus gracilis, mantidos por 5 dias em jejum alimentar. $\mathrm{N}=12$ indivíduos $\left(1^{\circ}\right.$ dia $=$ animais alimentados $)$

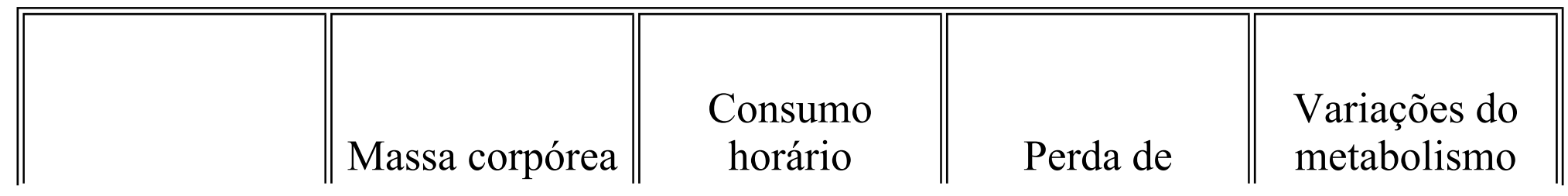




\begin{tabular}{||c||c||c||c||c||} 
Dias & $\begin{array}{c}\text { média } \pm \text { d.p } \\
(\mathrm{g})\end{array}$ & $\begin{array}{c}\text { média } \pm \text { d.p. } \\
\left(\mu 1 \mathrm{O}_{2} \cdot \mathrm{h}^{-1}\right)\end{array}$ & $\begin{array}{c}\text { Massa corpórea } \\
(\%)\end{array}$ & $(\mathbf{\%})$ \\
\hline \hline $1^{\mathbf{o}}$ & $0,0629 \pm 0,0070$ & $28,062 \pm 3,880$ & 100,00 & 100,00 \\
\hline \hline $2^{\mathbf{o}}$ & $0,0597 \pm 0,0066$ & $25,147 \pm 3,449$ & 94,91 & 89,61 \\
\hline \hline $3^{\mathbf{o}}$ & $0,0569 \pm 0,0064$ & $21,794 \pm 1,725$ & 90,46 & 77,66 \\
\hline \hline $4^{\mathbf{o}}$ & $0,0531 \pm 0,0061$ & $17,435 \pm 0,862$ & 84,42 & 62,13 \\
\hline \hline $5^{\mathbf{o}}$ & $0,0494 \pm 0,0057$ & $13,367 \pm 0,431$ & 78,54 & 47,63 \\
\hline \hline
\end{tabular}



Figura 4. Comparação entre massa corpórea e consumo de oxigênio a $25^{\circ} \mathrm{C}$ em um grupo de 12 indivíduos de Oxidus gracilis, mantidos por 5 dias em jejum alimentar $\left(1^{\circ}\right.$ dia $=$ animais alimentados).(Valores médios conforme tabela 1).

As relações entre metabolismo e tamanho em diplópodos, tal como obtidas neste estudo, têm sido analisadas por outros pesquisadores. MORRISON (1946) revelou que no diplópodo do gênero Julus havia uma proporcionalidade entre o metabolismo e a massa elevada ao expoente $2 / 3$ ou 0,66 . DWARAKANATH \& JOB $(1965 ; 1966)$ destacaram a estreita relação entre metabolismo, tamanho e a perda de água por transpiração na espécie indiana Spirostreptus asthenes. Estas relações também foram comprovadas em outra espécie indiana, Arthrosphaera dalyi, por PAULPANDIAN (1966). BYZOVA (1967) relatou que de 7 espécies de diplópodos da Rússia, em apenas uma (Sarmantiulus kessleri) não havia ajustamento entre o metabolismo e o tamanho. Em 15 espécies de artrópodos terrestres, entre as quais 4 espécies de diplópodos, REICHLE (1968) determinou um expoente $\mathbf{b}=0,84$, intermediário entre 0,66 e 1,00. O valor de $\mathbf{b}$ 
aqui calculado para Oxidus gracilis foi, portanto, muito parecido com os índices encontrados por REICHLE (1968) e foi também muito semelhante ao obtido por GROMYSZ-KALKOWSKA \& STOJALOWSKA (1966) para o diplópodo Orthomorpha gracilis, igualmente de origem tropical, mas adaptado em estufas de plantas na Polônia (b $=0,848)$.

A intenção dos pesquisadores de verificar em que proporção o metabolismo de um animal se relaciona com o tamanho, deriva da antiga "lei da superfície" em animais homeotermos proposta por Rubner \& Richet (conforme MENDES, 1953), onde as trocas de calor entre a superfície corpórea e o ambiente relacionavam-se com a massa elevada ao expoente 2/3 (3/4, segundo KLEIBER, 1932; 1947). Todavia, nos poiquilotermos, que são desprovidos de mecanismos de produção de calor corpóreo, $\mathrm{O}$ expoente de massa (valor de b) situa-se dentro da mesma variação encontrada em mamíferos (HEMMINGSEN, 1960; MENDES; 1953; SCHMIDT-NIELSEN, 1972). ZEUTHEN (1953) confirmou este fato obtendo os seguintes valores de b para animais em geral: 0,70 para organismos unicelulares, 0,95 para pequenos metazoários e 0,75 para poiquilotermos maiores e vertebrados homeotermos (aves e mamíferos). Considerando, entretanto, que o valor de b pode variar interespecificamente, BERTALANFFY (1957) propôs 3 tipos de metabolismo para os diferentes animais: num primeiro tipo o metabolismo seria proporcional à superfície ou à potência $2 / 3$ da massa corpórea, incluindo-se aí os peixes e alguns invertebrados (crustáceos e bivalvos); num segundo tipo o metabolismo seria proporcional à massa $(\mathbf{b}=1)$, onde se incluiriam as larvas de insetos em crescimento e certos hemimetábolos e, num terceiro tipo, o metabolismo ficaria entre a massa e a superfície corpórea e aí se incluiriam os caracóis de água doce, os turbelários e a maioria dos vertebrados. Outras correlações de metabolismo (valores de b) foram anteriormente sugeridas por LUDWIG (1956) e LUDWIG \& KRYWIEWCZYK (1950), que levaram em conta os tipos de mecanismos respiratórios: valores de $\mathbf{b}$ indicariam dependência da superfície em respiradores branquiados (bivalvos, caracóis prosobrânquios, isópodos aquáticos); uma dependência da massa em respiradores traqueados (artrópodos terrestres) e caracóis pulmonados; ou de ambos, em animais com duas rotas respiratórias, como tegumento e traquéias (Dixippus), brânquias e pseudo-brânquias (Porcellio). Essas subdivisões em categorias metabólicas, todavia, não explicam satisfatoriamente o por que da relação entre metabolismo e tamanho em animais. Assim, uma das explicações que encontram respaldo na literatura (MENDES \& ULIAN, 1987) é a admissão de WEIMOUTH et. al (1944), segundo o qual o consumo de oxigênio dos animais varia alometricamente com o tamanho, sempre que durante o crescimento houver um aumento desproporcionado de tecidos de baixa taxa metabólica, como gordura, tecido conjuntivo, tecido ósseo, etc. Esta interpretação estaria amparada na antiga proposição de Kestner apud ZEUTHEN (1947), que denominou de paraplasma as substâncias "não respirantes" e de protoplasma as "respirantes" do organismo. Assim, quanto maior a relação paraplasma/protoplasma, maior a massa metabolicamente inativa e menor a taxa metabólica. No caso dos diplópodos esta deve ser a razão fundamental, já 
que, com o aumento do tamanho aumenta também a massa metabolicamente inativa do exoesqueleto de quitina impregnado de sais de cálcio, fazendo reduzir, proporcionalmente, a taxa metabólica. Seria também uma das razões de serem, em geral, tão baixas as taxas respiratórias em diplópodos pois, "somente alguns celenterados sedentários mostram tão baixos consumos de oxigênio" (PAULPANDIAN, 1966).

Os valores de b listados na Tabela 2 para diplópodos, onde também se incluem os dados de Oxidus gracilis, ocupam uma ampla escala indo desde 0,1 até 1,2. Como em geral, a temperatura, as estações do ano, as latitudes geográficas ou as diferenças sexuais não seriam responsáveis pelas aparentes variações desses índices, talvez as diferenças pudessem ser explicadas pelas metodologias experimentais empregadas, ou pelas variações de gama de tamanho utilizadas nas medições. Contudo, a tendência de b ficar ao redor de 0,6 permitiria um comentário final em termos de mecanismos respiratórios dos diplópodos. Assim, considerando-se que a tomada de oxigênio se faz através das 4 aberturas das bolsas traqueais para cada diplossegmento do corpo (PENTEADO et al., 1991), isto poderia sugerir que o metabolismo respiratório de diplópodos dependeria, mais da superfície que da massa corpórea, tendo em vista a grande extensão da superfície respiratória exposta. Este fato, poderia contrariar as teorias de LUDWIG (1956) e LUDWIG \& KRYWIEWCZYK (1950), de acordo com as quais a respiração dos animais traqueados se relacionaria com a massa corpórea. Entretanto não se poderia descartar a possibilidade da relação entre o metabolismo e o tamanho dos diplópodos e de outros animais, ser também influenciada por outros fatores, tais como, genéticos, evolutivos, hormonais, grau de atividade e até mesmo a concentração do citocromo $\mathrm{C}$ que decresceria com o aumento da massa corpórea e com a idade dos indivíduos.

\section{Tabela 2 - Estimativa dos valores de $b$ e influência do sexo no consumo de oxigênio em diferentes diplópodos. Temp. 18-25oC.}

\begin{tabular}{|c|c|c|c|c|c|c|}
\hline Diplópodos & $\begin{array}{l}\text { Temp. } \\
\text { oC }\end{array}$ & $\begin{array}{c}\text { Classe de } \\
\text { tamanho } \\
(\mathrm{g})\end{array}$ & n & $\boldsymbol{b}$ & $\begin{array}{l}\text { Influência } \\
\text { do sexo }\end{array}$ & Referências \\
\hline $\begin{array}{l}\text { Orthomorpha } \\
\text { gracilis }\end{array}$ & 25 & $\begin{array}{c}0,031- \\
0,093\end{array}$ & 122 & 0,848 & não & \begin{tabular}{|l} 
GROMYSZ- \\
KALKOWSKA \& \\
STOJALOWSKA \\
$(1966)$
\end{tabular} \\
\hline \begin{tabular}{|l|} 
Chromatoiulus \\
rossicus
\end{tabular} & 18 & $\begin{array}{l}0,055- \\
0,230 \\
\end{array}$ & 52 & 0,500 & não & BYZOVA (1967) \\
\hline $\begin{array}{l}\text { Polydesmus } \\
\text { complanatus }\end{array}$ & 25 & $\begin{array}{c}0,050- \\
0,114\end{array}$ & 157 & 0,831 & não & $\begin{array}{l}\text { GROMYSZ- } \\
\text { KALKOWSKA } \\
(1970)\end{array}$ \\
\hline \begin{tabular}{|l} 
Spirostreptus \\
asthenes
\end{tabular} & 25 & $\begin{array}{l}2,000- \\
18,000\end{array}$ & --- & 0,681 & -- & $\begin{array}{l}\text { DWARAKANATH } \\
(1971)\end{array}$ \\
\hline
\end{tabular}




\begin{tabular}{|c|c|c|c|c|c|c|}
\hline $\begin{array}{l}\text { Pachyiulus } \\
\text { flavipes }\end{array}$ & 20 & $\begin{array}{l}0,010- \\
3,200 \\
\end{array}$ & 295 & 0,630 & --- & |BYZOVA (1971) \\
\hline Glomeris connexa & 22 & $\begin{array}{c}0,030- \\
0,140\end{array}$ & 139 & 0,660 & não & \begin{tabular}{|l} 
GROMYSZ- \\
KALKOWSKA \& \\
STOJALOWSKA \\
$(1971)$
\end{tabular} \\
\hline \begin{tabular}{|l} 
Polydesmus \\
gracilis
\end{tabular} & 20 & --- & --- & --- & $\operatorname{sim}$ & $\begin{array}{l}\text { DWARAKANATH, } \\
\text { BERLIN \& } \\
\text { PANDIAN (1972) }\end{array}$ \\
\hline $\begin{array}{l}\text { Strongylosoma } \\
\text { pallipes }\end{array}$ & 25 & $\mid \begin{array}{ll}0 & , 036-0 \\
0 & , 056\end{array}$ & $\begin{array}{l}34(\mathrm{~m}) \\
32(\mathrm{f})\end{array}$ & $\begin{array}{l}1,259 \\
1,102\end{array}$ & não & \begin{tabular}{|l} 
GROMYSZ- \\
KALKOWSKA \\
$(1973)$
\end{tabular} \\
\hline $\mid$\begin{tabular}{||l} 
Polydesmus \\
complanatus
\end{tabular} & 22 & $\begin{array}{l}0,052- \\
0,086\end{array}$ & 26 & 0,875 & não & \begin{tabular}{|l} 
GROMYSZ- \\
KALKOWSKA \& \\
STOJALOWSKA \\
$(1973)$
\end{tabular} \\
\hline $\begin{array}{l}\text { Arthrosphaera } \\
\text { disticta }\end{array}$ & 25 & $\begin{array}{l}1,000- \\
4,000\end{array}$ & 8 & 0,165 & --- & $\begin{array}{l}\text { DWARAKANATH, } \\
\text { BERLIN \& } \\
\text { PANDIAN (1972) }\end{array}$ \\
\hline Glomeris connexa & 25 & $\begin{array}{l}0,030- \\
0,130\end{array}$ & --- & $0,687(a)$ & --- & $\begin{array}{l}\text { GROMYSZ- } \\
\text { KALKOWSKA } \\
(1974)\end{array}$ \\
\hline \begin{tabular}{|l} 
Orthoporus \\
ornatus
\end{tabular} & 25 & $\begin{array}{l}0,287- \\
11,255\end{array}$ & 36 & 0,760 & não & $\begin{array}{l}\text { WOOTEN \& } \\
\text { CRAWFORD } \\
(1974)\end{array}$ \\
\hline $\mid \begin{array}{l}\text { Polyzonium } \\
\text { germanicum }\end{array}$ & 25 & $\begin{array}{l}0,008- \\
0,040\end{array}$ & $259(\mathrm{t})$ & $0,936(a)$ & não & $\begin{array}{l}\text { GROMYSZ- } \\
\text { KALKOWSKA } \\
(1976)\end{array}$ \\
\hline \begin{tabular}{|l} 
Rhinocricus \\
padbergi
\end{tabular} & 25 & $\begin{array}{l}0,084- \\
3,031 \\
\end{array}$ & 100 & 0,660 & não & $\begin{array}{l}\text { PENTEADO \& } \\
\text { MENDES (1977) }\end{array}$ \\
\hline \begin{tabular}{|l} 
Leptoiulus $p$. \\
proximus
\end{tabular} & 25 & $\begin{array}{l}0,090- \\
0,198\end{array}$ & $212(t)$ & $0,937(a)$ & não(s) & $\begin{array}{l}\text { GROMYSZ- } \\
\text { KALKOWSKA } \\
(1979)\end{array}$ \\
\hline $\begin{array}{l}\text { Ommatoiulus } \\
\text { Sabulosus }\end{array}$ & 20 & $\begin{array}{l}0,100- \\
0,302\end{array}$ & 187 & $\begin{array}{c}0,972- \\
1,000\end{array}$ & --- & $\begin{array}{l}\text { GROMYSZ- } \\
\text { KALKOWSKA, } \\
\text { TRACZ \& } \\
\text { SZUBARTOWSKA } \\
(1986)\end{array}$ \\
\hline \begin{tabular}{|l} 
Plusioporus \\
setiger
\end{tabular} & 25 & 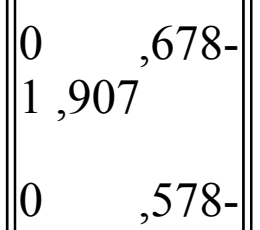 & $\begin{array}{l}24(\mathrm{~m}) \\
45(\mathrm{f})\end{array}$ & $\begin{array}{l}0,470 \\
0,360\end{array}$ & não & |PENTEADO (1986) \\
\hline
\end{tabular}




\begin{tabular}{|c|c|c|c|c|c|c|}
\hline & & 2,059 & & & & \\
\hline $\mid \begin{array}{l}\text { Ordem } \\
\text { Craspedosomida }\end{array}$ & $22-30$ & 0,012 & 24 & 0,769 & não & $\begin{array}{l}\text { HUMPHREYS \& } \\
\text { COLLIS (1990) }\end{array}$ \\
\hline Ordem Julida & 25 & 0,046 & 24 & 0,630 & não & \begin{tabular}{|l} 
HUMPHREYS \& \\
COLLIS (1990)
\end{tabular} \\
\hline $\begin{array}{l}\text { Pseudonannolene } \\
\text { tricolor }\end{array}$ & 25 & $\begin{array}{l}0,242- \\
2,802\end{array}$ & $\begin{array}{l}37(\mathrm{~m}) \\
53(\mathrm{f})\end{array}$ & $\begin{array}{l}0,680 \\
0,600\end{array}$ & não & $\begin{array}{l}\text { PENTEADO, } \\
\text { BERALDO \& } \\
\text { MENDES (1991) }\end{array}$ \\
\hline \begin{tabular}{|l} 
Gymnostreptus \\
olivaceus
\end{tabular} & 25 & $\begin{array}{l}0,160- \\
3,460 \\
\end{array}$ & 76 & 0,715 & não & $\begin{array}{l}\text { BOCCARDO \& } \\
\text { PENTEADO (1995) }\end{array}$ \\
\hline Oxidus gracilis & 25 & $\begin{array}{c}0,041- \\
0,083\end{array}$ & 45 & 0,86 & não & Este trabalho \\
\hline
\end{tabular}

$(\mathrm{m})=$ Machos, $(\mathrm{f})=$ Fêmeas; $(\mathrm{a})=$ Média para primavera, verão, outono e inverno; $(\mathrm{t})=$ Total de indivíduos usados na primavera, verão, outono e inverno;

$(s)=\operatorname{sim}$ na primavera.

Nos animais, as influências sexuais são condicionadas geneticamente e se traduzem hormonalmente, acentuando-se nos períodos reprodutivos (SILVA, 1997). Em diplópodos, as diferenças metabólicas entre machos e fêmeas ocorreram nas espécies americanas Pleuroloma flavipes butleri e Euryurus leachii (HALL et al., 1969) e na espécie indiana Polydesmus gracilis (DWARAKANATH et al., 1972), nas quais o metabolismo das fêmeas foi maior que o dos machos. $\mathrm{Na}$ espécie polonesa Leptoiuuls proximus proximus (GROMYSZ-KALKOWSKA, 1979 o metabolismo dos machos foi maior que o das fêmeas, porém, durante a fase reprodutiva, na primavera. No caso de Oxidus gracilis os valores obtidos para o expoente b $(0,89$ para os machos e 0,78 para as fêmeas) não foram significativamente diferentes entre si. Vale lembrar, porém, que tanto em Oxidus gracilis como na grande maioria dos diplópodos estudados (Tabela 2) as pesquisas foram realizadas em períodos de inatividade sexual, fato que poderia mascarar possíveis alterações metabólicas devidas ao sexo.

Os efeitos do jejum no metabolismo respiratório de diplópodos têm sido estimados em termos de variação das taxas respiratórias durante alguns dias. Em Schyzophylum sabulosum, BYZOVA (1967) verificou que após alguns dias de estivação, a taxa respiratória cai cerca de 50\%. Em Polydesmus complanatus e Orthomorpha gracilis (GROMYSZ-KALKOWSKA, 1970) as maiores reduções na taxa respiratória ocorreram no $1^{\circ}$ dia de jejum: 16,8 e 9,6\%, respectivamente. Em Glomeris connexa (GROMYSZ-KALKOWSKA \& STOJALOWSKA, 1971) a redução, após o $1^{\circ}$ dia sem alimento foi de $13,9 \%$, mas a maior redução ocorreu no $3^{\circ}$ dia: $33,8 \%$. Em Strongylosoma pallipes (GROMYSZ-KALKOWSKA, 1973) foi registrada a maior redução na taxa respiratória após 24 horas de jejum: 21,6\%. A autora sugeriu que a intensa atividade locomotora de $S$. pallipes seria a causa provável para esta redução tão 
acentuada. BYZOVA (1972), todavia, registrou em Pachyulus flavipes uma dupla redução em 2 dias de observação, seguida de aumento na respiração, explicado como uma necessidade de restabelecer os recursos hídricos. Em Rhinocricus padbergi (PENTEADO \& MENDES, 1977) um jejum de 5 dias não afetou significativamente o consumo horário de oxigênio e as condições fisiológicas do animal, mas causou uma perda de massa corpórea de cerca de 33\%. Fatos semelhantes foram observados em Pachydesmus crassicutis (STEWART \& WOODRING, 1973) em situações similares de temperatura e umidade, tendo-se constatado que o animal perde $6,05 \%$ de massa por dia, retornando após 5 dias ao terrário em boas condições. Em Oxidus gracilis, os experimentos aqui realizados mostraram que este diplópodo também retornou aos terrários em boas condições fisiológicas, mas sofreu maior redução da taxa metabólica do que da massa do corpo. Neste aspecto Oxidus gracilis apresentou um resultado inverso do verificado com Rhinocricus padbergi.

\section{REFERÊNCIAS BIBLIOGRÁFICAS}

BAILEY, N.T.J. Statistical methods in Biology. London: The English University Press Ltd., 1959. 199p.

BERTALANFFY, L.V. Quantitative laws in metabolism and growth. Quarterly Rev. Biol., v. 32, n. 3, p. 217-31, 1957.

BOCCARDO, L. Fisioecologia do diplópodo Gymnostreptus olivaceus Schubart, 1944 (Spirostreptida, Spirostreptidae): Termopreferência, atividade e metabolismo respiratório. Dissertação (Mestrado em Zoologia), Instituto de Biociências , UNESP - Rio Claro, SP, 1992. 113 p.

BOCCARDO, L. Ecofisiologia do diplópodo Gymnostreptus olivaceus Schubart, 1944 (Spirostreptida, Spirostreptidade). Metabolismo respiratório e tolerâncias às variações de temperatura e tensão de oxigênio. Tese (Doutorado em Zoologia), Instituto de Biociências, UNESP, Rio Claro, SP, 1996. 94 p.

BOCCARDO, L, PENTEADO, C.H.S. Preferências Térmicas e respostas metabólicas em relação à temperatura e ao tamanho em Gymnostreptus olivaceus Schubart, 1944 (Diplopoda, Spirostreptida). Rev. Bras. Biol., v.3, n.55, 445-56, 1995.

BRITES, V.L.C. Preferências térmicas, atividade e metabolismo respiratório na barata Blaberus piracicabensis Piza, 1960 (Dictyoptera, Blaberidae). Dissertacão (Mestrado em Zoologia), Instituto de Biociências, UNESP, Rio Claro, SP, 1982. 144 p.

BYZOVA, J.B. Respiratory metabolism in some millipedes (Diplopoda). Rev. Ecol. 
Biol. Sol., v. 4, n. 4, p. 611 - 24, 1967.

BYZOVA, J.B. Oxygen consumption with respect to weight in Pachyiulus flavipes (Diplopoda, Julidae). Zool. Zh. (URSS), v.60 ,n.11, p.1737-40, 1971.

BYZOVA, J.B. Oxygen consumption in millipedes (Diplopoda, Julidae) in the period of enforced starvation. Zool. Zh. (URSS), v.51, n.3, p.364-9, 1972.

DIXON, M. Manometric Methods. Cambridge Univ. Press \& New York: MacMillan, 1943. $155 \mathrm{p}$.

DWARAKANATH, S.K. The influence of body size and temperature upon the oxigen consumption in the millipede, Spirostreptus asthenes (Pocock). Comp. Biochem. Physiol., v.38A, p.351 - 58, 1971.

DWARAKANATH, S.K., JOB, S.V. Studies on transpiration in millipedes. I. Spirostreptus asthenes Poc., from a tropical jungle near Madurai. Proc. Indian. Acad. Sci., v.61, n.3, p.142-6, 1965.

DWARAKANATH, S.K., JOB, S.V. Studies on transpiration in millipedes. II. Respiration and water loss in Spirostreptus asthenes. Proc. Ind. Acad. Sci., v.62B, n.5, p.224 - 8, 1966.

DWARAKANATH, S.K., BERLIN, O.G.W., PANDIAN, R.S. Respiratory metabolism in Polydesmus gracilis (Diplopoda) as a function of temperature and sex. Curr. Sci., v.41, n.5, p.184-5, 1972.

DWARAKANATH, S.K., BERLIN, O.G.W., PANDIAN, R.S. Oxygen consumption as a function of size and temperature in pill millipede Arthrosphaera disticta (Pocock). Monit. Zool. Ital., v.7, p. 43 - 50., 1973.

GRANER, E.A. Estatística. São Paulo: Edições Melhoramentos, 1966. 184 p.

GROMYSZ-KALKOWSKA, K. The influence of body weigth, external temperature, seasons of the year and fasting on respiratory metabolism in Polydesmus complanatus L. (Diplopoda). Folia Biol. (Kracow), v.18, n.4, p.311 - 26, 1970.

GROMYSZ-KALKOWSKA, K. Some exogenous and endogenous effects on rate of respiration in Strongylosoma pallipedes (Olivier) (Diplopoda) and behavior in temperature gradient. Folia Biol. (Kracow), v.21, n. 3, p.251 - 69, 1973.

GROMYSZ-KALKOWSKA, K. The effect of some exogenous factors and body weight on oxygen consumption in Glomeris connexa C.L. Kock (Diplopoda). Folia. Biol.(Kracow), v.27, n.1, p.37-49, 1974. 
GROMYSZ-KALKOWSKA, K. The oxygen consumption of Polyzonium germanicum Brdt. (Diplopoda) in relation to some exogenous and endogenous factors. Folia Biol.(Kracow), v. 24, n.4, p.401 - 15, 1976.

GROMYSZ-KALKOWSKA, K. Some properties of the respiratory metabolism of the adult Leptoiulus proximus proximus (Nem.) (Diplopoda). Folia Biol. (Kracow), v.27, n.2, p.129 - 45, 1979.

GROMYSZ-KALKOWSKA, K. \& STOJALOWSKA, W. Respiratory metabolism in Orthomorpha gracilis. C.L. Koch (Diplopoda) as a function of temperature and body size. Folia Biol. (Kracow), v.14, n.4, p. 379 - 89, 1966.

GROMYSZ-KALKOWSKA, K. \& STOJALOWSKA, W. Oxygen consumption level in Glomeris connexa $\mathrm{C}$. $\mathrm{L}$. Koch (Diplopoda) in different physiolological states. Folia Biol. (Kracow), v.19, n.4, p.457 - 72, 1971.

GROMYSZ-KALKOWSKA, K. \& STOJALOWSKA, W. Respiration rates of eggs, larval stages, and adult individuals of Polydesmus complanatus (L.) (Diplopoda). Folia Biol. (Kracow), v.21, n.3, p. 271 - 8, 1973.

GROMYSZ-KALKOWSKA, K., TRACZ, H., SZUBARTOWSKA, E. Some aspects of respiration rate in Ommatouilus sabulosus (L.). Folia Soc. Sci. Lublinensis Biol., v.28, p.65-76, 1986.

HALL, F.R., HOLLINGWORTH, R.M., SHANKLAND, D.L. Cyanide tolerance in millipedes: comparison of respiration in millipedes and insects. Entomol. News, v.80, n.11, p.277-82, 1969.

HEMMINGSEN, A.M. Energy metabolism as related to body size and respiratory surfaces and its evolution. Resp. Steno Mem. Hosp. Copenh. v.9 (Part 2), p.1-110, 1960.

HUMPHREYS, W.F., COLLIS, G. Water loss and respiration of cave arthropods from Cape Range, Western Australia. Comp. Biochem. Physiol., v.95 A , n.1, p.1017, 1990 .

HUXLEY, J.S. Problems of relative growth. London: Methuen and Co. , 1932. 166p. 1932.

KLEIBER, M. Body size and metabolism. Hilgardia, v.6, n.11, p. 313 - 53, 1932.

KLEIBER, M. Body size and metabolic rate. Physiol. Rev., v.27, p.511-41, 1947.

LUDWIG, W. Bertrachtung über den Energiekonsum von Tieren mit 
Atmungsorganen von Zweierlei typ. Z. vergl. Physiol., v.39, p. 84 - 8, 1956.

LUDWIG, W., KRYWIENCZYK, J. Köpergrösse, Körperzeiten und Energiebilanz. III: Mitteilung Der Sauerstoffverbrauch von Muscheln in Abbãngigkeit von der Körgrözze. Z. vergl. Physiol., v.32, p.464-7, 1950.

MENDES, E.G. Metabolismo, tamanho e latitude. Ci. e Cult., v.5, n.1, p. 13 - 8, 1953.

MENDES, E.G., ULIAN, G.B. The influence of size, temperature and oxygen tension upon the respiratory metabolism of the terrestrial amphipod Talitrus (Talitroides) pacificus Hurley, 1955. Comp. Biochem. Physiol., v.86A, p.155-62, 1987.

MORRISON, P.R. Physiological observations on water loss and oxygen consumption in Peripatus. Biol. Bull. v.91, p.181-8, 1946.

NIELSEN, C.O. Respiratory metabolism of some populations of enchytraeid worms and freeliving nematodes. Oikos, v.12, n.1, p. 17- 35, 1961.

PAULPANDIAN, A. A note on the respiration of the pill millipede Arthrosphaera dalyi (Pocock). Curr. Sci., v.7, p.178-9, 1966.

PENTEADO, C.H.S. Tolerância, atividade e respiração no diplópode Rhinocricus padbergi Verhoeff, 1938. Tese (Doutorado em Ciências). Faculdade de Filosofia, Ciências e Letras de Rio Claro, SP, 1972. 197p.

PENTEADO, C.H.S. Metabolismo respiratório no diplópodo Plusioporus setiger (Broelemann, 1902) (Diplopoda, Spirostreptidae): Os Fatores Tamanho e Sexo. Ci. e Cult. V.38, n.9, p.1587 - 93, 1986.

PENTEADO, C.H.S., MENDES, E.G. Respiratory metabolism and tolerance in a tropical millipede, Rhinocricus padbergi Verhoeff, 1938.I The structure of the tracheal pocket and the respiratory rate at normoxic conditions and $25^{\circ} \mathrm{C}$. Rev. Bras. Biol., v.37,n.2., p. 431 - 46, 1977.

PENTEADO, C.H.S., MENDES, E.G. Respiratory metabolism and tolerance in a tropical millipede, Rhinocricus padbergi Verhoeff, 1938. III: The responses to temperature variations. Rev. Bras. Biol., v.41, n.3, p. 499 - 509, 1981.

PENTEADO, C.H.S., HEBLING-BERALDO, M.J.A \& MENDES, E.G. Oxygen consumption related to size and sex in the tropical millipede Pseudonannolene tricolor (Diplopoda, Spirostreptida). Comp. Biochem. Physiol., v.98 A, n.2, p.265-9, 1991. 
PHILLIPSON, J. Ecological Energetics. London: Edward Arnold Ltd., 1966. 57p.

PROSSER, C.L. Comparative Animal Physiology: I. Environmental Physiology. $3^{\text {rd }}$ ed., Philadelphia: W.B. Saunders Co., 1973. 454p.

REICHLE, D.E. Relation of body size to food intake, oxygen consumption and trace element metabolism in forest floor arthopods. Ecology, v.49, n.3, p.539 - 42, 1968.

SCHMIDT-NIELSEN, K. How animals work. Capt. 6. Body size and problems of scaling. Cambridge: Cambridge Univ. Press, 1972, p.85-109.

SCHUBART, O. Os diplópodos de Pirassununga. Acta Zoológica Lilloana del Instituto "Miguel Lillo", v. 2, p.321-440, 1944.

SCHUBART, O. Gongolôs, emboás ou diplópodos. Ciênc. e Cult., v.7, n.4, p.214-20, 1955.

SILVA, R. S. Fisioecologia do diplópodo Oxidus gracilis (Koch, 1847) (Polydesmida, Strongylosomidae). Respostas comportamentais e metabólicas. Dissertação (Mestrado em Zoologia), Instituto de Biociências (UNESP), Rio Claro, 1997, 93p.

STAMOU, G.P., IATROU, G.D. Studies on the respiratory metabolism of Glomeris balcanica (Diplopoda, Glomeridae). J. Insect. Physiol., v.39, n.6, p.529-35, 1993.

STEWART, T.C., WOODRING, J.P. Anatomical and physiological studies of water balance in the millipedes Pachydesmus crasicustis (Polydesmida) and Orthoporus texticolens (Spirobolida). Comp. Biochem. Physiol., v.44A, p.735-60, 1973

WEIMOUTH, F.W.; CRISMON, J.M., HALL, V.E., BELDING, H.S. \& FIELD, J. Total and tissue respiration in relation to body weight. Physiol. Zool., v.17, p.50-71, 1944.

WOOTEN, R.C., CRAWFORD, C.S. Respiratory metabolism of the desert millipede Orthophorus ornatus (Girard) (Diplopoda). Oecologia, v.17, p.179-86, 1974.

ZEUTHEN, E. Body size and metabolic rate in animal kingdon with special regard to the marine microfauna. Compt. Rend. lab. Carlsberg. Sér. chim., v.26, p.20-161, 1947.

ZEUTHEN, E. Oxygen uptake as related to body size in organisms. Quart. Rev. Biol., v.28, p.1-12, 1953. 
Os autores agradecem à CAPES, ao Departamento de Zoologia - I.B.UNESP - Rio Claro, ao Centro de Estudos Ambientais (CEA-UNESP- Rio Claro) e ao Dr. Richard L. Hoffman, do Virginia Museum of Natural History, Virgínia - USA, pela identificação do material. 